\title{
DPT/Typhoid/Staphylococcus aureus/Paratyphoid A/Paratyphoid B Vaccine
}

National Cancer Institute

\section{Source}

National Cancer Institute. DPT/Typhoid/Staphylococcus aureus/Paratyphoid

A/Paratyphoid B Vaccine. NCI Thesaurus. Code C120214.

A proprietary lipid emulsion containing five vaccines: diphtheria, pertussis, tetanus (DPT), typhoid, Staphylococcus aureus, paratyphoid A and paratyphoid B, with potential immunostimulating activity. Subcutaneous administration of the DPT/typhoid/Staphylococcus aureus/paratyphoid A/paratyphoid B vaccine activates the immune system and may both abrogate tumor-induced immune tolerance and induce an antitumor immune response, which may eradicate the tumor. 